\title{
Effects of physical and sporting activities on balance control in elderly people
}

\author{
Philippe P Perrin, Gérome C Gauchard, Cyril Perrot, Claude Jeandel
}

Unité de Formation et de Recherche en Sciences et Techniques des Activités Physiques et Sportives, Université Henri Poincaré - Nancy 1, F 54600

Villers-lès-Nancy, France

P P Perrin

G C Gauchard

C Perrot

Laboratoire d'Exploration

Fonctionnelle, Service

ORL, Centre

Hospitalier

Universitaire de

Nancy, F 54500

Vandoeuvre-lès-Nancy,

France

P P Perrin

Inserm U420, Faculté de Médecine de Nancy, F 54 500,

Vandoeuvre-lès-Nancy, France

P P Perrin

Service de Médecine Interne B,

Gérontologie, Centre

Hospitalier

Universitaire de

Nancy, F 54500

Vandoeuvre-lès-Nancy,

France

C Jeandel

Correspondence to:

Prof Ph Perrin, Equilibration et Performance Motrice,

UFR STAPS, Université

Henri Poincaré - Nancy 1,

30 , rue du Jardin Botanique,

54600 Villers-lès-Nancy,

France.

Accepted for publication 21 December 1998

\begin{abstract}
Objective-Balance disorders increase with aging and raise the risk of accidental falls in the elderly. It has been suggested that the practice of physical and sporting activities (PSA) efficiently counteracts these age related disorders, reducing the risk of falling significantly.

Methods-This study, principally based on a period during which the subjects were engaged in PSA, included 65 healthy subjects, aged over 60, who were living at home. Three series of posturographic tests (static, dynamic with a single and fast upward tilt, and dynamic with slow sinusoidal oscillations) analysing the centre of foot pressure displacements or electromyographic responses were conducted to determine the effects of PSA practice on balance control.
\end{abstract}

Results-The major variables of postural control were best in subjects who had always practised PSA (AA group). Those who did not take part in PSA at all (II group) had the worst postural performances, whatever the test. Subjects having lately begun PSA practice (IA group) had good postural performances, close to those of the AA group, whereas the subjects who had stopped the practice of PSA at an early age (AI group) did not perform as well. Overall, the postural control in the group studied decreased in the order AA $>$ IA $>$ AI $>$ II.

Conclusions-The period during which PSA are practised seems to be of major importance, having a positive bearing on postural control. It seems that recent periods of practice have greater beneficial effects on the subject's postural stability than PSA practice only at an early age. These data are compatible with the fact that PSA are extremely useful for elderly people even if it has not been a lifelong habit.

(Br f Sports Med 1999;33:121-126)

Keywords: posture; elderly; training; posturography

Balance requires a contribution from three areas-namely, information provided by balance sensors (visual, vestibular, and somatosensory), central integration in the brain, and motor response. Failure of postural control under adverse environmental factors, in association with the alteration of this tripartite system owing to aging, might be responsible for the falls of elderly people. ${ }^{1}$ These falls, a real problem in public health, are the main cause of accidental death in the elderly. ${ }^{2}$ Decline in postural control is influenced by inactivity, ${ }^{3}$ yet several studies have shown that the practice of physical and sporting activities (PSA) at various levels of skill improves postural performances ${ }^{5-7}$ and reduces, as a consequence, the number of falls. ${ }^{8-10}$ However, these studies were all based on setting up a training programme for a group and comparing this group with an untrained control group, or on a comparison of several training programmes. Our study examined balance control for four groups of older adults, grouped according to the period during which they had practised PSA. We wanted to examine the influence of continuous PSA practice on postural control in the elderly, and to determine whether it is beneficial for an elderly person to start a physical activity programme at an age when he/she might not be motivated to begin exercising.

\section{Subjects and methods}

SUBJECTS

Sixty five retired subjects aged over 60 who were living at home were recruited from a cohort of elderly subjects taking part in a larger study on age related physiology. They were contacted by telephone and agreed to come to the centre housing posturographic facilities.

The subjects comprised 43 women and 22 men, aged between 60 and 85 (mean (SE) 71.8 (0.8)) who all lived at home and could perform their daily tasks without help. Moreover, they were free from any disease of the central nervous system and did not show any orthopaedic disorder either of the trunk or the lower limbs as assessed previously in the larger study mentioned above. All had been examined by bone densitometry, had had an ear, nose and throat examination, and psychometric evaluation, confirming their overall good health. All subjects had comparable body mass indices.

Before the balance and posturography tests were performed - that is, without knowledge of the outcome of these tests, a questionnaire and a complementary interview were used to define four groups according to the period during which PSA were practised. None of the subjects tested was or had been a professional sportsperson. The physical activities reported had been or were practised for leisure. The groups were as follows:

- The first group comprising 13 subjects (10 women, 3 men, mean age 73.9) who had practised PSA all their life, with only a minor interruption, if any, was called "active-active" (AA). They still practised at least one sporting activity (walking, swimming, cycling, or tennis). 


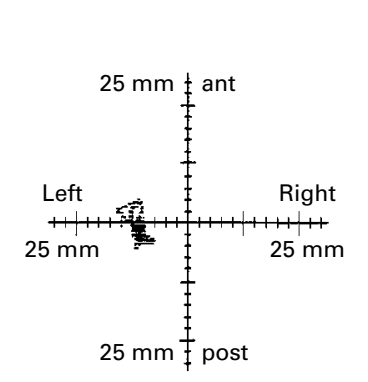

\section{Sweep}

Eyes open

$\begin{array}{ll}\text { Sway path } & : 0.60 \mathrm{~cm} / \mathrm{s} \\ \text { Area } & : 0.24 \mathrm{~cm}^{2} / \mathrm{s} \\ \text { Ant-post/lat } & : 1.66\end{array}$

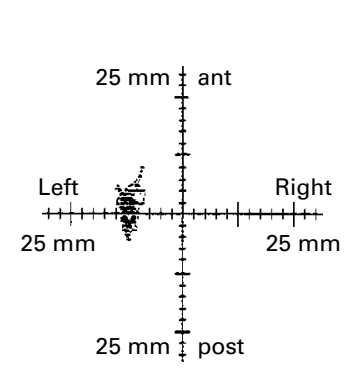

2. Sweep

Eyes closed

\section{Sway path $\quad: 0.76 \mathrm{~cm} / \mathrm{s}$ \\ Area $\quad: 0.36 \mathrm{~cm}^{2} / \mathrm{s}$ \\ Ant-post/lat $: 3.08$}

Figure 1 Statokinesigram recordings from static tests performed with eyes open (1) or closed (2). Graphs on the left show the whole recording of the centre of foot pressure displacements and provide sway path (indicated as way for the distance covered) and area (for the surface covered). Graphs on the right are a vectorial analysis of centre of foot pressure displacements, providing the amplitude of anteroposterior and lateral sway. This figure is a typical data sheet provided by the platform's software.

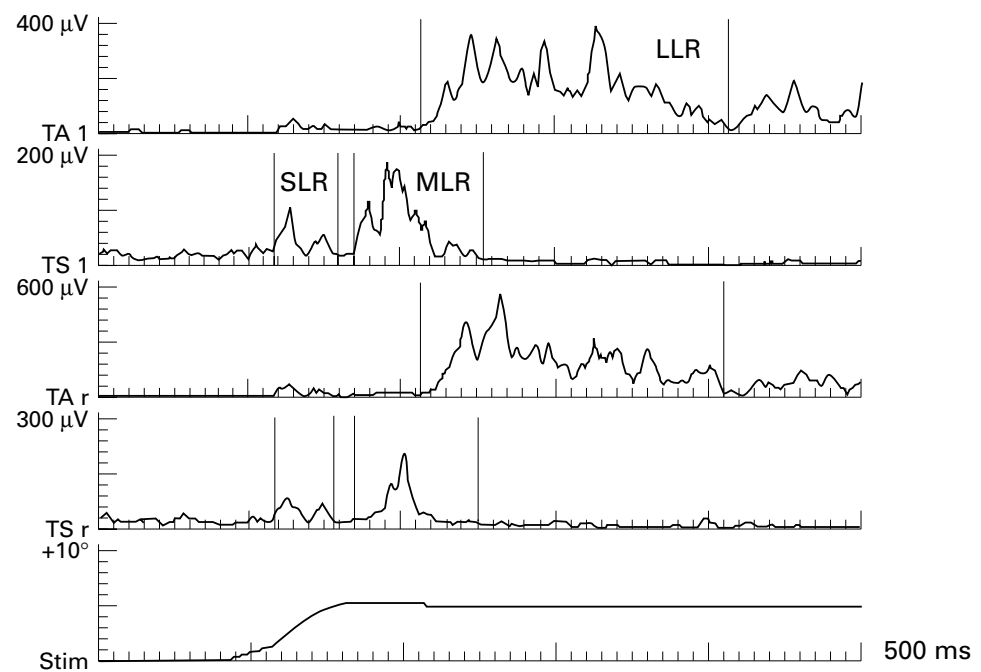

Figure 2 Dynamic test with sudden toes-up platform tilt: electromyographic recordings, from the tibialis anterior muscle (TA) and the gastrocnemius medialis of the triceps surae muscle (TS). Stimulus: platform movement. According to the time elapsed since the stimulus (abscissa), three responses can be measured as short latency response (SLR), medium latency response (MLR); long latency response (LLR) expressed in microvolts (ordinate).

- The second group comprising 25 people (17 women, 8 men, mean age 71.9 ) who began PSA practice after retiring was considered "inactive-active" (IA). This group practised two major activities-walking for $85 \%$ of them and/or soft gymnastics, such as yoga, for $80 \%$. Other activities, such as swimming and cycling, were practised respectively by $40 \%$ and $15 \%$ of
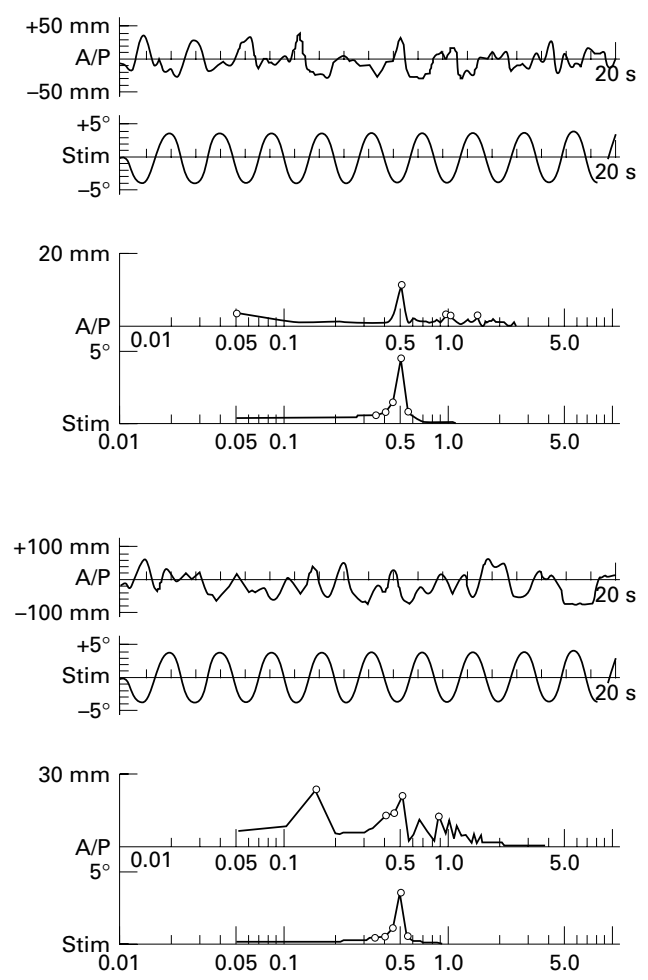

Figure 3 Centre of foot pressure displacements recorded during a sinusoidal posturographic test with oscillations in the sagittal plane. Sinusoidal platform movements (Stim). Typical recordings of centre of foot pressure displacements (anteroposterior $(A / P)$ ) and their fast Fourier transformation. The abscissa indicates the time elapsed during the graphic recordings and the frequency in Hertz for fast Fourier transformation graphs. Amplitudes, on the ordinate scales, are expressed in millimetres for subjects' movements and degrees for the platform's movement. Type I recordings are homogeneous curves; type II recordings are inhomogeneous curves, characteristically irregular and representative of higher instability.

the people in this group, which also included a semimarathon runner.

- The third group comprising 14 people (9 women, 5 men, mean age 67.9) who had practised PSA during their youth, and definitely stopped at least 30 years ago, was described as "active-inactive" (AI). These subjects had been cycling in $70 \%$ of the cases and walking in $60 \%$ of the cases. Some of them had practised such activities as basketball, cross country skiing, and swimming. None had been forced to stop owing to vertigo or serious trauma.

- The fourth group comprising 13 subjects ( 7 women, 6 men, mean age 73.7 ) who had never practised PSA was called "inactiveinactive" (II).

\section{METHODS}

All subjects underwent three posturographic tests (static, fast dynamic, and slow sinusoidal dynamic) on a vertical force platform (Tönnies $\mathrm{GmbH}$, Freiburg, Germany), fitted with four pressure gauges located in the corners, from which the centre of foot pressure positions were recorded as well as its displacements. These tests were performed in a soundproof room with artificial lightning.

For each test the subject was required to remain as stable as possible but relaxed, standing barefoot on the platform, arms along the 

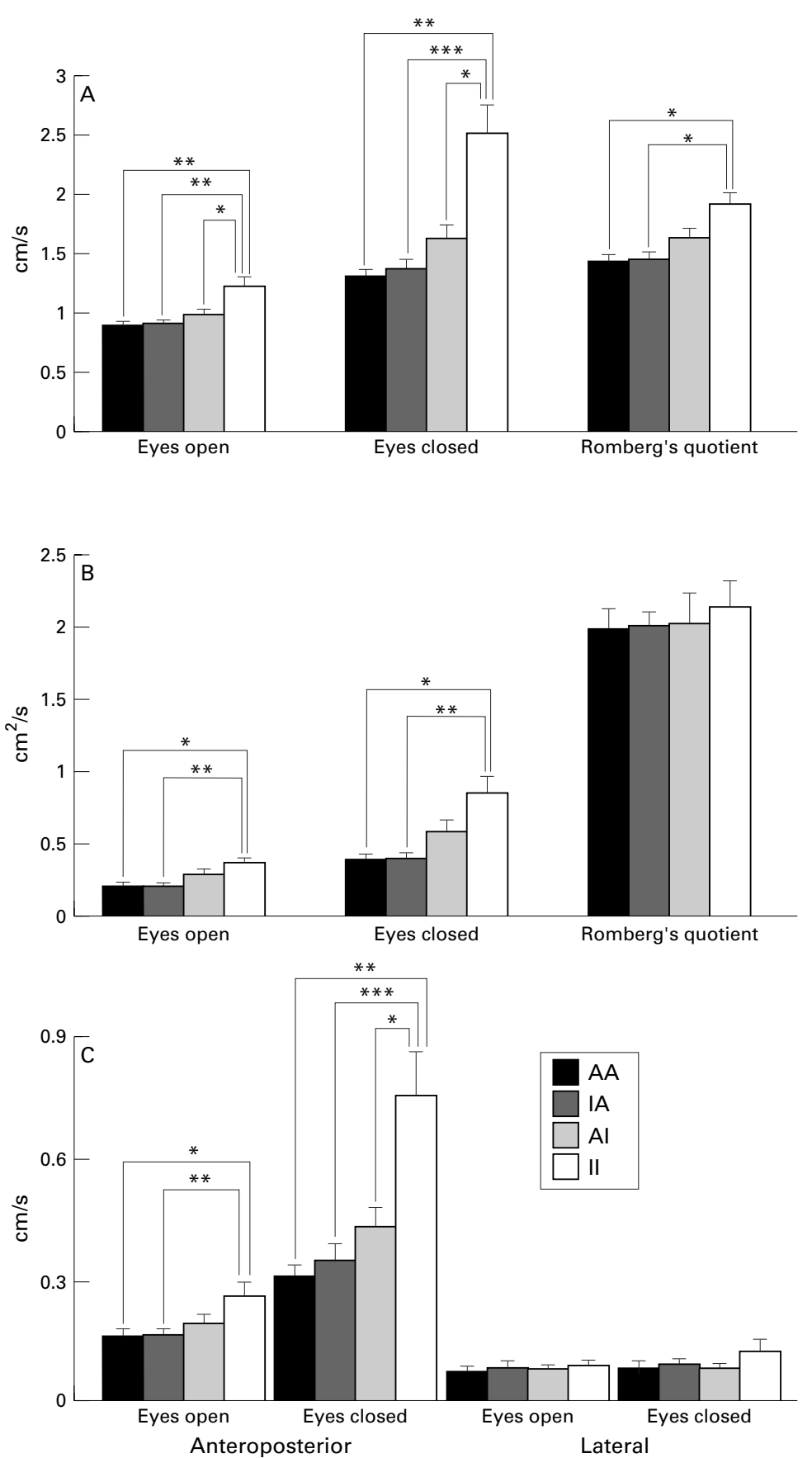

Figure 4 Comparative results of statokinesigram recordings in those who had always practised physical and sporting activities (PSA) (group AA), subjects having lately begun PSA practice (group IA), subjects who had stopped the practice of PSA at an early age (group AI), and those who did not take part in PSA at all (group II) in tests performed with eyes open or closed; the SE is also given. Results are expressed as distance travelled (cm) per time (s) or sway path/s (cm) (graph A), or area $\left(\mathrm{cm}^{2}\right)$ per time (s) or area/s $\left(\mathrm{cm}^{2}\right)$ (graph B), and anteroposterior and lateral oscillations $(\mathrm{cm})$ per time (s) (graph C). Statistical significances are indicated as follows: ${ }^{\star} p<0.05 ;{ }^{\star} p<0.01 ;{ }^{\star \star \star} p<0.001$.

body and breathing normally, fixing a mark horizontally placed on a wall $2 \mathrm{~m}$ away.

Static posturographic tests were performed for 20 seconds with the eyes open, then for 20 seconds with the eyes closed, and the displacement of the centre of foot pressure was recorded. The statokinesigrams obtained allowed measurement of the sway path and the area covered by the centre of foot pressure movements and the sway in anteroposterior and lateral axes (fig 1). Romberg's quotient- eyes closed/eyes open sway ratio-was calculated; this determines the importance of the visual contribution. ${ }^{11} 12$

During the first dynamic posturographic test ${ }^{13}$ the subjects were submitted to eight consecutive and unexpected toes-up movements generated by a single and rapid tilt of the platform ( $4^{\circ}$ amplitude, velocity $50^{\circ} / \mathrm{s}$ ). Electromyographic recordings of the anterior tibial muscle and medial head of the gastrocnemius muscle of the triceps surae were simultaneously performed on the left and the right legs. Latency and amplitude of the responses (fig 2) were averaged from the eight successive tests performed for each subject.

Three EMG responses were obtained: (a) the short latency response, collected on the triceps surae as an M-shaped double peak, corresponding to a myotatic reflex; (b) the medium latency response, identified in $90 \%$ of the cases, also recorded on the triceps surae, indicating a polysynaptic reflex; (c) the long latency response, obtained on the anterior tibial muscle, contributing to secure stabilisation. ${ }^{14} 15$

During the second dynamic posturographic test the movement imposed by the platform consisted of slow sinusoidal anteroposterior oscillations with a $4^{\circ}$ amplitude at a $0.5 \mathrm{~Hz}$ frequency. ${ }^{14}$ This test lasted 20 seconds and was performed with eyes open and closed. Centre of foot pressure movements were recorded during the test and also expressed as a fast Fourier transformation. Figure 3 shows the two types of recording obtained, representing different sensorimotor strategies ${ }^{16}{ }^{17}$ according to different responses to destabilisation $^{18}$ : (a) type I recordings, characterised by regular patterns, at the same frequency as the stimulus, but in opposite phase with low or high amplitude, are considered to be homogeneous, indicating great stability of the subject during the test; (b) type II recordings, characterised by irregular patterns, at a different frequency from that of the stimulus, with high amplitude in the low frequencies, are considered to be inhomogeneous, and convey the instability of the subject during the recording. ${ }^{18} 19$

Falls were defined by observing the subject and noting when he/she reached for support or was obviously leaning on the security belt.

\section{STATISTICS}

All subjects were able to perform all the tests. Results in each group were expressed as means (SE) between groups. Comparisons were performed for the static and fast dynamic tests using analysis of variance, and with a $\chi^{2}$ test for the slow dynamic tests using Statview software.

\section{Results}

\section{STATIC TEST}

Figure 4 shows the results for the sway path and area parameters, Romberg's quotient, and results for the anteroposterior and lateral sways.

The data obtained with the eyes closed were worse than those obtained with the eyes open in all groups. 


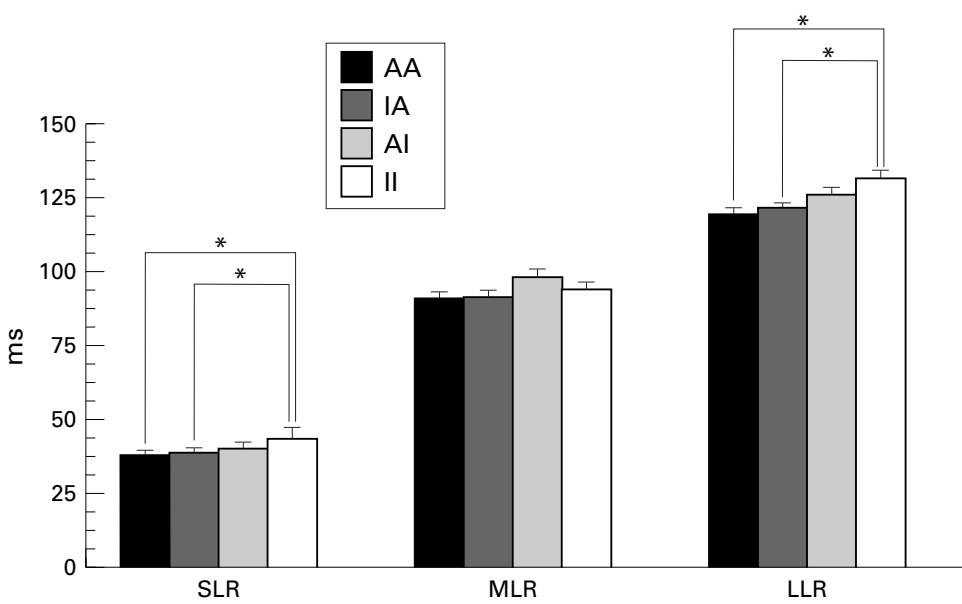

Figure 5 Dynamic test with sudden toes-up platform tilt. Comparative results of short $(S L R)$, medium $(M L R)$, and long latency responses (LLR) electromyographic recordings in groups $A A, I A, A I$, and II; the $S E$ is also shown. (See fig 4 for definition of groups.) Statistical significances are indicated as follows: ${ }^{*} p<0.05 ;{ }^{* *} p<0.01$.

Table 1 Dynamic test with sudden toes-up platform tilt. Amplitudes of the short (SLR), medium $(M L R)$, and long latency responses (LLR) electromyographic recordings in the four groups of subjects studied. Results are shown as median (range)

\begin{tabular}{|c|c|c|c|}
\hline Group * & $S L R(\mu V)$ & $M L R(\mu V)$ & $L L R(\mu V)$ \\
\hline$\overline{\mathrm{AA}}$ & $74(32-182)$ & $110(40-233)$ & $327(145-604)$ \\
\hline IA & $80(21-862)$ & $69(24-1085)$ & $375(70-892)$ \\
\hline $\mathrm{AI}$ & $85(16-842)$ & $113(10-756)$ & $399(96-1381)$ \\
\hline II & $100(13-409)$ & $88(26-660)$ & $355(95-1190)$ \\
\hline
\end{tabular}

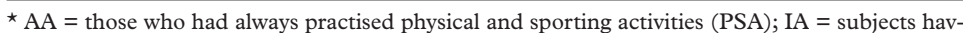
ing lately begun PSA practice; $\mathrm{AI}=$ subjects who had stopped the practice of PSA at an early age; II $=$ those who did not take part in PSA at all.

Higher sway path values were seen with a decrease in sports practice (eyes open: AA 0.89 (0.19) $\mathrm{cm} / \mathrm{s}$, II $1.23(0.46) \mathrm{cm} / \mathrm{s})$, the differences being statistically significant between all sports practice groups (AA, IA, and AI) and the group that did not practise sport (II) both with eyes open and closed $(p<0.05)$.

Higher area values were obtained with the decrease of sports practice (eyes closed: AA $0.39(0.22) \mathrm{cm}^{2} / \mathrm{s}$, II $\left.0.85(0.75) \mathrm{cm}^{2} / \mathrm{s}\right)$ with statistically significant differences between the groups currently practising PSA (AA and IA), and the non-sports group (II), both with eyes open and eyes closed $(\mathrm{p}<0.05)$.

Romberg's quotient for the centre of foot pressure displacements area was similar in all groups, but the quotient for the centre of foot displacements sway path was significantly lower in both practising groups (AA and IA) than in the II group (AA $1.43(0.24)$, II 1.92 $(0.7))(\mathrm{p}<0.05)$.

Higher anteroposterior sway values were seen with a decrease in sports practice (eyes closed: AA $0.31(0.12) \mathrm{cm} / \mathrm{s}$, II $0.75(0.61)$ $\mathrm{cm} / \mathrm{s}$ ) and the differences were significant between currently practising groups (AA and IA), and the non-sports group (II) whether the eyes were open or closed $(\mathrm{p}<0.01)$. The AI group did not differ from the II group when the eyes were open, but significant differences were found when the eyes were closed $(\mathrm{p}<0.05)$.

FAST DYNAMIC TEST

Short latency responses were higher in the inactive group (AA 38.2 (2.9) $\mathrm{ms}$, II 43.47 $(17.28) \mathrm{ms}$ ), the differences being significant between the currently practising groups (AA
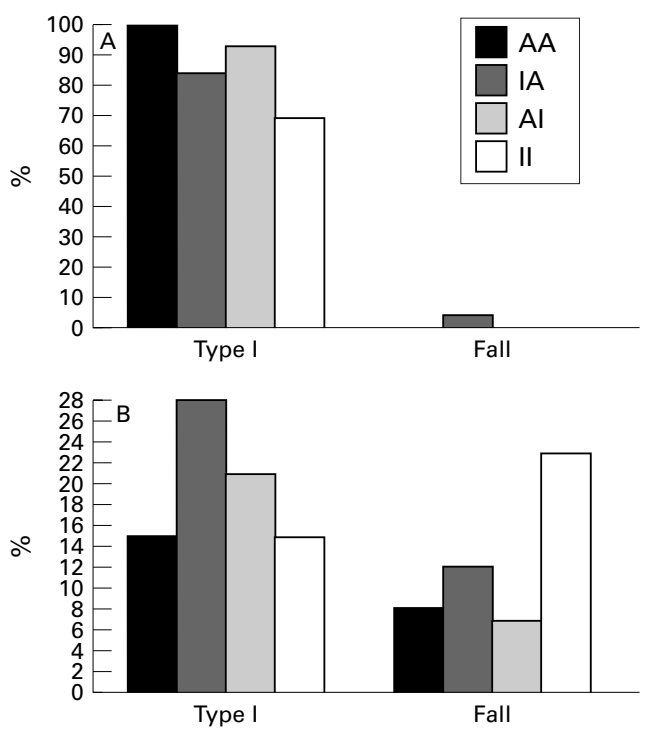

Figure 6 Comparative percentages of subjects displaying type I patterns during a sinusoidal posturographic test in groups $A A, I A, A I$, and II, in tests performed with eyes open $(A)$ or eyes closed (B). (See fig 4 for definition of groups.)

and IA) and the II group ( $\mathrm{p}<0.05$ ) (fig 5). Long latency responses were higher in the inactive group (AA 120.4 (7.32) ms, II 130.92 (16.2) $\mathrm{ms}$ ), the differences being significant between the currently practising groups (AA and IA) and the II group $(\mathrm{p}<0.05)$ (fig 5$)$.

There was no significant difference in the amplitude of the short and long latency response, though there seemed to be a tendency towards higher values with lower PSA practice, except in the inactive group (table 1).

SLOW SINUSOIDAL TEST

There was no statistically significant difference between the groups during the slow sinusoidal test. All groups mainly had homogeneous patterns with eyes open, with slightly more type II recordings in the II group (fig 6A). Conversely, inhomogeneous type II patterns were predominant with eyes closed for all the groups (AA $78 \%$, IA $60 \%$, AI $72 \%$, II $62 \%$ ). There were also several falls with the eyes closed (AA 8\%, IA $12 \%$, AI $7 \%$ ), especially in the II group (23\%) (fig 6B).

\section{Discussion}

This study reports significant differences in posture control between elderly subjects practising PSA and those who are not.

The static test allows an evaluation of body sway and of the role of the visual contribution. Good static postural control, which declines with age,${ }^{18}$ is characterised by a small sway path (conveying a small consumption of energy) and by a small area covered by the centre of foot pressure movements (conveying precision). ${ }^{20} 21$ Our results indicate that these parameters, whether with eyes open or eyes closed, are improved by exercise, and that recent practice is as beneficial as practice in the past, or permanent practice. Continuing practice of PSA might be more profitable ${ }^{72}$ because aging and a sedentary way of life are accompanied by 
a reduction of the muscular mass ${ }^{23}$ and strength, ${ }^{24}{ }^{25}$ which may be prevented or delayed by PSA. We can also infer that a period of exercise, even performed a long time ago (AI group), allows for postural control and a lower consumption of energy (sway path/s) for a similar precision (area/s) compared with the complete absence of PSA (II group). Alternatively, subjects with poor balance control might choose to avoid physical activities. However, none of the II or AI subjects reported poor balance as the reason why they did not start or had stopped PSA practice.

Several studies have shown that aging is accompanied by an increasing Romberg's quotient, indicating a greater importance of the visual information. ${ }^{26-28}$ Our results indicate that PSA practice decreases the dependency upon the visual input. This could be achieved by a better use of somatosensory inputs through PSA practice and improved balance control as their seemed to be an II $<\mathrm{AI}<\mathrm{IA}$ hierarchy. As the Romberg quotient values in the AI group were not statistically different from those in the II group, it may be suggested that exercise does not provide long term benefits for the substitution of vision by another contributing factor: the AI and II groups are more visually dependent than currently practising subjects. Comparable information has been provided in studies comparing sports beginners and experts, the latter being less dependent on a visual cue. ${ }^{29}$

Our results show that for all groups the anteroposterior sway is predominant, probably because of the orientation of leg joints in man. Sway increases with age owing both to strength loss of the ankle dorsiflexor muscles ${ }^{30}$ and to decreasing tactile sensitivity, joint position sense, and proprioception. ${ }^{31}{ }^{32}$ Sway was, however, smaller in currently practising subjects (AA and IA groups). This could be explained by an optimisation of somatosensory inputs, PSA increasing the ankles' muscular force and/or tactile sensitivity and proprioception. ${ }^{33}$ Proprioception and sensory input from the plantar surface of the feet have been reported to be the most important sensorial system for maintaining balance under normal conditions. $^{32}$ Physical activities, through the increased usage of these stimuli, seem to allow more efficient postural adaptation. This is consistent with the postural control deduced from our data, which in the groups studied decreased in the order $\mathrm{AA}>\mathrm{IA}>\mathrm{AI}>\mathrm{II}$.

The fast dynamic test allows evaluation of the nervous conduction and proprioceptive inputs. Several studies ${ }^{1834} 35$ indicate that one of the effects of aging is to reduce nervous conduction velocity. We observed shorter latencies of muscular contraction (short and long latency responses) in elderly subjects practising PSA. Similar trends have been shown in younger sportsmen, ${ }^{36}$ with current practice appearing to be the most profitable. This shorter latency observed in subjects practising PSA could reflect a more appropriate neuromuscular control. ${ }^{37}$

The slow dynamic test evaluates balance inputs and their interactions in balance control. Several studies using the same type of test have reported increased instability related to aging. ${ }^{18-38}$ In group II a larger number of inhomogeneous graphs were recorded both with eyes open and with eyes closed, though the differences did not reach statistical significance. A decrease of articular mobility, especially at the ankle, as well as a decline of the ability to resolve sensorial information conflicts, might be the cause of these poorer results, forcing group II subjects to use an unstable strategy, ${ }^{38}$ but these data should be confirmed in a larger series of subjects. Conversely, for the AA, IA, and AI groups, the achievement of more than $90 \%$ of homogeneous graphs during tests with the eyes open might suggest that exercise improves dynamic qualities and good control coordination. ${ }^{24} 39$ Moreover, with eyes closed, PSA seemed to be beneficial as most fallers belonged to the II group.

In conclusion, this study suggests, through the use of a combination of posturographic tests, that PSA have a positive effect on balance control in elderly subjects. As this study compared four groups of people, we can also conclude that it is of major importance to recommend PSA to the elderly, even if they never practised any earlier in their life, as no difference was seen between those who had begun PSA late in life and those who had either never stopped or practised PSA a long time ago.

\section{Contributors}

Philippe P Perrin planned the study, collected and interpreted data, wrote most of the drafts and final manuscript, and will act as guarantor. Gérome C Gauchard collected and interpreted the data, performed statistical analyses, discussed drafts, and approved the final manuscript. Cyril Perrot collected and interpreted the data, discussed drafts, and approved the final manupreted the data, discussed drafts, and approved the final manuassessment of the subjects and contributed to the paper.

1 Maki BE, Holliday PJ, Topper AK. Fear of falling and postural performance in the elderly. F Gerontol 1991;46:M12331 .

2 Overstall PW, Johnson AL, Exton-Smith AN. Instability and falls in the elderly. Age Ageing 1978;7(suppl):92-6.

3 Hindmarsh JJ, Estes EH. Falls in older persons. Arch Intern Med 1989;149:2217-22

4 Morris JN, Hardman AE. Walking to health. Sports Med 1997;23:306-32.

5 Lord SR, Castell S. Effect of exercise on balance, strength and reaction time in older people. Australian Physiotherapy 1994;40:83-8.

6 Wolfson L, Whipple R, Derby C, et al. Balance and strength training in older adults: intervention gains and Tai Chi maintenance. 7 Am Geriatr Soc 1996;44:498-506.

7 Buchner DM, Cress ME, de Lateur BJ, et al. A comparison of the effects of three types of endurance training on balance and other fall risk factors in older adults. Aging (Milano) 1997;9:112-19.

8 Province MA, Hadley EC, Hornbrook MC, et al. The effects of exercise on falls in elderly patients. A preplanned metaanalysis of the FICSIT trials. Frailty and Injuries: Cooperative Studies of Intervention Techniques. $7 A M A$ 1995;273:1341-7.

9 Wolf SL, Barnhart HX, Kutner NG, et al. Reducing frailty and falls in older persons: an investigation of Tai Chi and computerized balance training. Atlanta FICSIT Group. Frailty and Injuries: Cooperative Studies of Intervention Techniques. F Am Geriatr Soc 1996;44:489-97.

10 Campbell AJ, Robertson MC, Gardner MM, et al. Randomised controlled trial of a general practice programme of home based exercise to prevent falls in elderly women. BMF 1997;315:1065-9.

11 Van Parys JAP, Njiokiktiien ChJ. Romberg's sign expressed in a quotient. Agressologie 1976;17B:95-100.

12 Tropp H, Odenrick P, Sandlund B, et al. Stabilometry for studying postural control and compensation in vertigo of central and peripheral origin. Electromyogr Clin Neurophysiol 1987;27:77-82.

13 Diener HC, Bootz F, Dichgans J, et al. Variability of postural "reflexes" in humans. Exp Brain Res 1983;52:423-8.

14 Haas G, Diener HC, Bacher M, et al. Development of postural control in children: short-, medium- and long latency 
EMG responses of leg muscles after perturbation of stance. Exp Brain Res 1986;64:127-32.

15 Allum JHJ. Posturography systems: current measurement concepts and possible improvements. In: Brandt Th, concepts and possible improvements. In: Brandt Th, Paulus W, Bles W, et al, eds. Disorders of posture and gait. Stuttgart, New York: Thieme, 1990:16-28.

16 Nashner LM. Strategies for organization of human posture. In: Igarashi M, Black FO, eds. Vestibular and visual control on posture and locomotor equilibrium. Basel: Karger, 1985:1-8.

17 Horak FB, Nashner LM. Central programming of postural movements: adaptation to altered support-surface configuration. F Neurophysiol 1986;55:1369-81.

18 Perrin Ph, Jeandel C, Perrin C, et al. Influence of visual control, conduction and central integration on static and dynamic balance in healthy older adults. Gerontology 1997 43:223-31.

19 Perrin Ph, Béné MC, Perrin C, et al. Ankle trauma significantly impairs posture control: a study in basketball players and controls. Int $f$ Sports Med 1997;18:387-92.

20 Norré ME. Posture in otoneurology. Acta Otorhinolaryngol Belg 1990;44:363.

21 Toupet M, Gagey PM, Heuschen S. Vestibular patients and aging subjects lose use of visual input and expend more energy in static postural control. In: Vellas B, Toupet M Rubenstein L, et al, eds. Balance and gait disorders in the elderly. Paris: Elsevier, 1992:183-98.

22 Fiatarone MA, O’Neill EF, Ryan ND, et al. Exercise training and nutritional supplementation for physical frailty in very elderly people. N Engl F Med 1994;330:1769-75.

23 Lexel J. Human aging, muscle mass, and fiber type composition. F Gerontol 1995;50A:11-16.

24 Meusel H. Developing physical fitness for the elderly through sport and exercise. Br F Sports Med 1984;18:4-12.

5 Hurley BF. Age, gender, and muscular strength. F Gerontol 1995;50A:41-4

26 Pyykkö I, Aalto H, Hytonen $\mathrm{M}$, et al. Effect of age on postural control. In: Amblard B, Berthoz A, Clarac F, eds. Posture and gait: development, adaptation and modulation. Amsterdam, NewYork, Oxford: Excerpta Medica, 1988: Am-104.

27 Lafont Ch, Dupui Ph, Costes-Salon MC, et al. Equilibre et vieillissement. In: L'année gérontologique. Paris: Serdi, 1991: 151-91.
28 Straube A, Botzel K, Hawken M, et al. Postural control in the elderly: differential effects of visual, vestibular and somatosensory input. In: Amblard B, Berthoz A, Clarac F, eds. Posture and gait: development, adaptation and modulation. Amsterdam, New York, Oxford: Excerpta Medica, 1988: $105-14$.

29 Mesure S, Amblard B, Crémieux J. Effect of training on head-hip co-ordinated movements during unperturbed stance. Neuroreport 1997;8:3507-12.

30 Whipple RH, Wolfson LI, Amerman PM. The relationship of knee and ankle weakness to falls in nursing home resident: an isokinetic study. $\mathcal{F}$ Am Geriatr Soc 1987;35:1320.

31 Skinner HB, Barrack RL, Cook S. Age-related decline in proprioception. Clin Orthop 1984;184:208-11.

32 Lord SR, Clark RS, Webster IW. Postural stability and associated physiological factor in a population of aging persons. f Gerontol 1991;46:M69-76.

33 Nardone A, Giordano A, Corra T, et al. Responses of leg muscles in humans displaced while standing: effects of types of perturbation and of postural set. Brain 1990;113: $65-84$.

34 Woollacott MH, Shumway-Cook A, Nashner LM. Aging and posture control: changes in sensory organization and muscular coordination. Int $\mathcal{F}$ Aging Hum Dev 1986;23:97114

35 Woollacott $\mathrm{MH}$. Age-related changes in posture and movement. F Gerontol 1993;48(special issue):56-60.

$36 \mathrm{Perrin} \mathrm{Ph}$, Perrin $\mathrm{C}$, Courant $\mathrm{Ph}$, et al. Posture in basketball players. Acta Otorhinolaryngol Belg 1991;45:341-7.

37 Lord SR, Ward JA, Williams P. Exercise effect on dynamic stability in older women: a randomized controlled trial. Arch Phys Med Rehabil 1996;77:232-6.

38 Manchester D, Woollacott MH, Zederbauer-Hylton N, et al. Visual, vestibular and somatosensory contributions to balance control in older adult. 7 Gerontol 1989;44:M118-27.

39 Perrin $\mathrm{Ph}$, Schneider D, Deviterne $\mathrm{D}$, et al. Training improves the adaptation to changing visual conditions in maintaining posture control in a test of sinusoidal oscillation of the support. Neurosci Lett 1998;245:155-8.

\section{Take home message}

Postural control assessment performed in elderly subjects with various stories of physical and sporting activities (PSA) practice indicated that only those who had never practised PSA displayed significantly altered balance control. These data suggest that PSA, even when started late in life, have a positive effect on balance.

\section{Commentary}

Many functions of the human body deteriorate with age. Some of these events cannot be prevented, but a lot can be done about balance. The authors studied four groups of elderly people - one group physically active, one group inactive, one group who had stopped their sporting activities, and a fourth group that had become active after retirement from professional work. Using sophisticated balance measurements, they prove that activity increases balancing ability even if the sporting activities were started after the age of 60 . Inactivity led to a measured balance function at a lower level even if the person had been active with sports and physical activities earlier in life.

Several studies have been published showing that balance training is of great value. Increased muscle strength may be one cause for this improvement, but balance training alone without muscle strengthening also improves balancing ability. This study is important because it shows that physical activities improve the balancing ability, which of course is one way to prevent falls and fractures, especially in the elderly. 